\title{
On Bose-Einstein condensation on closed Robertson-Walker spacetimes
}

\author{
M. Trucks, \\ Institut für Theoretische Physik \\ Technische Universität Berlin \\ Hardenbergstraße 36, 10623 Berlin, Germany \\ E-mail: trucks@physik.tu-berlin.de
}

\begin{abstract}
In this letter we summarize our analysis of Bose-Einstein condensation on closed Robertson-Walker spacetimes. In a previous work we defined an adiabatic KMS state on the Weyl-algebra of the free massive Klein-Gordon field [1,2]. This state describes a free Bose gas on Robertson-Walker spacetimes. We use this state to analyze the possibility of Bose-Einstein condensation on closed Robertson-Walker spacetimes. We take into account the effects due to the finiteness of the spatial volume and show that they are not relevant in the early universe. Furthermore we show that a critical radius can be defined. The condensate disappears above the critical radius.
\end{abstract}

\section{Introduction}

We analyze the possibility of Bose-Einstein condensation of a free relativistic charged Bose gas on closed Robertson-Walker spacetimes. The free Bose gas is described by an adiabatic KMS state, which we defined in $[1,2]$. In the framework of algebraic quantum field theory on curved spacetimes (see [3]), we proved that an adiabatic KMS state is a Hadamard state, i.e. a physically relevant state. Furthermore, it describes a free Bose gas and the inverse temperature change was shown to be proportional to the scale parameter in the metric of the Robertson-Walker spacetime.

Bose-Einstein condensation is usually considered in the thermodynamic limit. Singularities appear in thermodynamic quantities, e.g. for a nonrelativistic Bose gas, there is a cusp-like singularity in the specific heat at the critical temperature. For a finite system, these singularities are smoothed out and there is no well-defined phase transition point. Furthermore, macroscopic occupation of the ground state sets in, although the temperature is higher than 
the critical temperature of the corresponding system in the thermodynamic limit, as we will see below.

We can apply known results about Bose-Einstein condensation on the Einstein universe (a closed Robertson-Walker spacetime with constant scale parameter) to closed Robertson-Walker spacetimes. This is justified on the one hand by the fact that adiabatic KMS states are Hadamard states and on the other hand because on every Cauchy surface an adiabatic KMS state on a closed Robertson-Walker spacetime has the form of a KMS state on an Einstein universe. Bose-Einstein condensation on the Einstein universe was analyzed by Singh and Pathria [4] and Parker and Zhang [5]. In the work of Singh and Pathria the emphasis is on the finite size effects, while they are not taken into account in the work of Parker and Zhang. In contrast the work of Parker and Zhang also gives some hints on Bose-Einstein condensation on closed Robertson-Walker spacetimes and a critical radius is defined. We analyze Bose-Einstein condensation on closed Robertson-Walker spacetimes, take into account the finite size effects and define a critical radius. If the Robertson-Walker spacetime expands, there is a radius, below which the gas is in a condensed state. The condensate disappears above this radius, but due to the finite size effects, this is not a well-defined phase transition point. Using realistic values for the early universe, we will see that the finite size effects are very small.

In the next section we calculate the charge density of the Bose gas on closed Robertson-Walker spacetimes. An approximation is made for the interesting regime, where the chemical potential reaches the value of the mass parameter. This formula is analyzed in section 3. A numerical calculation shows the effects of the finite spatial volume of closed Robertson-Walker spacetimes. The critical temperature of a charged relativistic Bose gas on Minkowski spacetime serves as a useful reference point, which enables us to define a critical radius. This critical radius depends only on the total charge and a constant $C=R T$, given by the product of the temperature and the scale parameter of the RobertsonWalker metric. Due to the finite spatial volume, there is a nonvanishing charge density in the ground state at the critical temperature (resp. radius). This value (scaled by the charge density) mainly depends on $C$.

\section{The charge density}

In this section we calculate the charge density of a relativistic charged Bose gas described by an adiabatic KMS state. An adiabatic KMS state on closed Robertson-Walker spacetimes can be defined on a Cauchy surface at time $t$ by 


$$
\omega(\cdot)=Z^{-1} \operatorname{tr}\{\exp [-\beta H(\mu)] \cdot\}
$$

where $H(\mu)$ is the second quantization of $h(\mu)=\left[m^{2}-\Delta / R^{2}(t)\right]^{1 / 2}-\mu, \beta$ is the inverse temperature, $\mu$ the chemical potential, $R$ the scale parameter in the metric of the Robertson-Walker spacetime, $Z=\operatorname{tr}\{\exp [-\beta H(\mu)]\}$ and $\Delta$ the Laplace operator on the three-sphere $S^{3}$, the spatial part of the spacetime. The conserved charge, which may be the electric or any other conserved charge, is described by the operator $Q=N_{+}-N_{-}$, where $N_{+}$resp. $N_{-}$are number operators for particles with positive resp. negative charge. Then the state is given by

$$
\omega(\cdot)=Z^{-1} \operatorname{tr}\{\exp [-\beta(H-\mu Q)] \cdot\}
$$

For the expectation value of the charge density in this state we have

$$
q=<Q>_{\beta, \mu} / V=\left(<N_{+}>_{\beta, \mu}-<N_{-}>_{\beta, \mu}\right) / V
$$

It is

$$
<N_{ \pm}>_{\beta, \mu}=\sum_{\vec{k}} \frac{\mathrm{e}^{ \pm \beta \mu}}{\mathrm{e}^{\beta \tilde{\omega}_{k}}-\mathrm{e}^{ \pm \beta \mu}}
$$

where $\tilde{\omega}_{k}=\sqrt{k(k+2) / R^{2}(t)+m^{2}}, k \in \mathbb{N}_{0}$, since $k(k+2)$ are the eigenvalues of the Laplace operator on the three-sphere. Using the fact that the multiplicity of the eigenvalues is $(k+1)^{2}$, we obtain

$$
q=\frac{2}{V} \sum_{j=1}^{\infty} \sinh (\beta \mu j) \sum_{k=1}^{\infty} k^{2} \mathrm{e}^{-\beta \omega_{k} n}
$$

where $\omega_{k}=\sqrt{\tilde{m}^{2}+k^{2} / R^{2}}, \tilde{m}^{2}=m^{2}-1 / R^{2}$. The sums can be simplified with the Poisson summation formula

$$
\sum_{k=1}^{\infty} f(k)=\int_{0}^{\infty} f(t) d t+\sum_{p=1}^{\infty} \int_{0}^{\infty} f(t) \cos (2 \pi p t) d t
$$

The following computation follows [4]. The first integral is given in [6, 3.389 Nr.4] and we have to assume $R>1 / m$. To solve the second integral, we expand the cosine in a power series and use the formula

$$
\sum_{l=0}^{\infty} \frac{\left(z \tau^{2} / 2\right)^{l}}{l !} \frac{K_{l+\lambda}(z s)}{s^{l+\lambda}}=\frac{K_{\lambda}\left(z \sqrt{s^{2}-\tau^{2}}\right)}{\left(s^{2}-\tau^{2}\right)^{\lambda / 2}}
$$


(see $[7,7.15 \mathrm{Nr} .9])$, to obtain

$$
\int_{0}^{\infty} f(t) \cos (2 \pi p t) d t=j \beta \tilde{m}^{4} R^{3}\left(\frac{K_{2}(a \tau)}{(a \tau)^{2}}-(2 \pi p \tilde{m} R)^{2} \frac{K_{3}(a \tau)}{(a \tau)^{3}}\right)
$$

where $\tau=\sqrt{(j \beta / R)^{2}+(2 \pi p)^{2}}, a=\tilde{m} R$ and $K_{\nu}(x)$ are modified Bessel functions. Therefore

$$
q=\frac{\tilde{m}^{3}}{2 \pi^{2}} W(\beta, \mu)+\frac{2 \beta \tilde{m}^{4}}{\pi^{2}} \sum_{j, p=1}^{\infty} j \sinh (j \beta \mu)\left(\frac{K_{2}(a \tau)}{(a \tau)^{2}}-(2 \pi p a)^{2} \frac{K_{3}(a \tau)}{(a \tau)^{3}}\right)
$$

where we defined $W(\beta, \mu)=2 \sum_{j=1}^{\infty}(j \beta \tilde{m})^{-1} \sinh (j \beta \mu) K_{2}(j \beta \tilde{m})$. We can also handle the sum over $j$ with the Poisson summation formula in the form

$$
\begin{aligned}
\sum_{j=1}^{\infty} f(j) & =\frac{1}{2} \sum_{j=-\infty}^{\infty} f(j)=\sum_{p=-\infty}^{\infty} \int_{-\infty}^{\infty} f(j) \cos (2 \pi p j) d j \\
& =\frac{1}{2} \sum_{p=-\infty}^{\infty} \Re \int_{0}^{\infty} j \sinh \left(j \beta \mu^{\prime}\right)\left(\frac{K_{2}(a \tau)}{(a \tau)^{2}}-(2 \pi p \tilde{m} R)^{2} \frac{K_{3}(a \tau)}{(a \tau)^{3}}\right) d j
\end{aligned}
$$

see [4, App.] where $\mu^{\prime}=\mu+2 \pi \mathrm{i} p / \beta$ and $\Re$ denotes the real part. The result is

$$
q=\frac{\tilde{m}}{2 \pi^{2}} W(\beta, \mu)-\frac{1}{\pi \beta} \Re\left\{\sum_{l=-\infty}^{\infty} \frac{\mu^{\prime} \sqrt{\tilde{m}^{2}-\mu^{\prime 2}}}{\exp \left(2 \pi R \sqrt{\tilde{m}^{2}-\mu^{\prime 2}}\right)}\right\},
$$

The second term comes from the finiteness of the spatial volume of the system. It can be shown that all terms with $l \neq 0$ can be neglected if $R / \beta \gg 1$, an inequality surely satisfied in the early universe. The term with $l=0$ is the significant term, giving the main contribution especially if $\mu \rightarrow \tilde{m}$. So we have

$$
q=\frac{\tilde{m}}{2 \pi^{2}} W(\beta, \mu)-\frac{\mu}{\pi^{2} \beta R} \frac{\pi R \sqrt{\operatorname{m} \tilde{m}^{2}-\mu^{2}}}{\exp \left(2 \pi R \sqrt{\tilde{m}^{2}-\mu^{2}}\right)-1} .
$$

The charge density in the ground state is given by

$$
q_{0}=\frac{1}{2 \pi^{2} R^{3}}\left(\left(\mathrm{e}^{\beta(m-\mu)}-1\right)^{-1}-\left(\mathrm{e}^{\beta(m+\mu)}-1\right)^{-1}\right) .
$$

If $\mu \rightarrow m$, we approximate $q_{0}$ by 


$$
\begin{aligned}
q_{0} & \approx \frac{1}{2 \pi^{2} R^{3}}\left([\beta(m-\mu)]^{-1}-[\beta(m+\mu)]^{-1}\right) \\
& =\frac{1}{\beta \pi^{2} R^{3}} \frac{\mu}{m^{2}-\mu^{2}} \\
& =\frac{\mu}{R \beta\left(y^{2}+\pi^{2}\right)}
\end{aligned}
$$

where we defined the thermogeometric parameter $y=\pi R \sqrt{\tilde{m}^{2}-\mu^{2}}$ (see [4]). The charge density in the ground state grows rapidly if $y^{2} \rightarrow-\pi^{2}$.

If $\mu \rightarrow m$ we can expand the function $W(\beta, \mu)$ (see $[4,8])$ :

$$
\frac{\tilde{m}^{3}}{2 \pi^{2}} W(\beta, \mu)=\frac{\tilde{m}^{3}}{2 \pi^{2}} W(\beta, \tilde{m})-\frac{\tilde{m}}{2 \pi \beta} \sqrt{\tilde{m}^{2}-\mu^{2}}+O\left(\tilde{m}^{2}-\mu^{2}\right),
$$

which leads to

$$
q \approx \frac{\tilde{m}^{3}}{2 \pi^{2}} W(\beta, \tilde{m})-\frac{\tilde{m}}{2 \pi^{2} R \beta} y \operatorname{coth} y \approx \frac{m^{3}}{2 \pi^{2}} W(\beta, m)-\frac{m}{2 \pi^{2} R \beta} y \operatorname{coth} y
$$

\section{The finite size effects}

We have to solve formula (1) for the unknown value $\mu$. This can only be done numerically. It is well-known that for finite systems there is no welldefined critical temperature. The critical temperature of the system in the thermodynamic limit can only serve as a useful reference point. For the system in the thermodynamic limit, i.e. for Minkowski spacetime, the critical (inverse) temperature is given by

$$
q=q\left(\beta_{c}, m\right)=\frac{m^{3}}{2 \pi^{2}} W\left(\beta_{c}, m\right)
$$

Using this reference point, we get as an equation for $y$ and also for $\mu$,

$$
y \operatorname{coth} y=-\frac{2 \pi^{2} R q \beta}{m}\left(1-\frac{W\left(\beta_{c}, m\right)}{W(\beta, m)}\right),
$$

which can be used to determine the charge density in the ground state $q_{0}$. We have used this equation to analyze the finite size effects. This is shown in fig. 1. It can be seen clearly that there is no well-defined phase transition point. Enlarging the radius $R$ forces the curve for the Einstein universe towards the curve for Minkowski spacetime. 


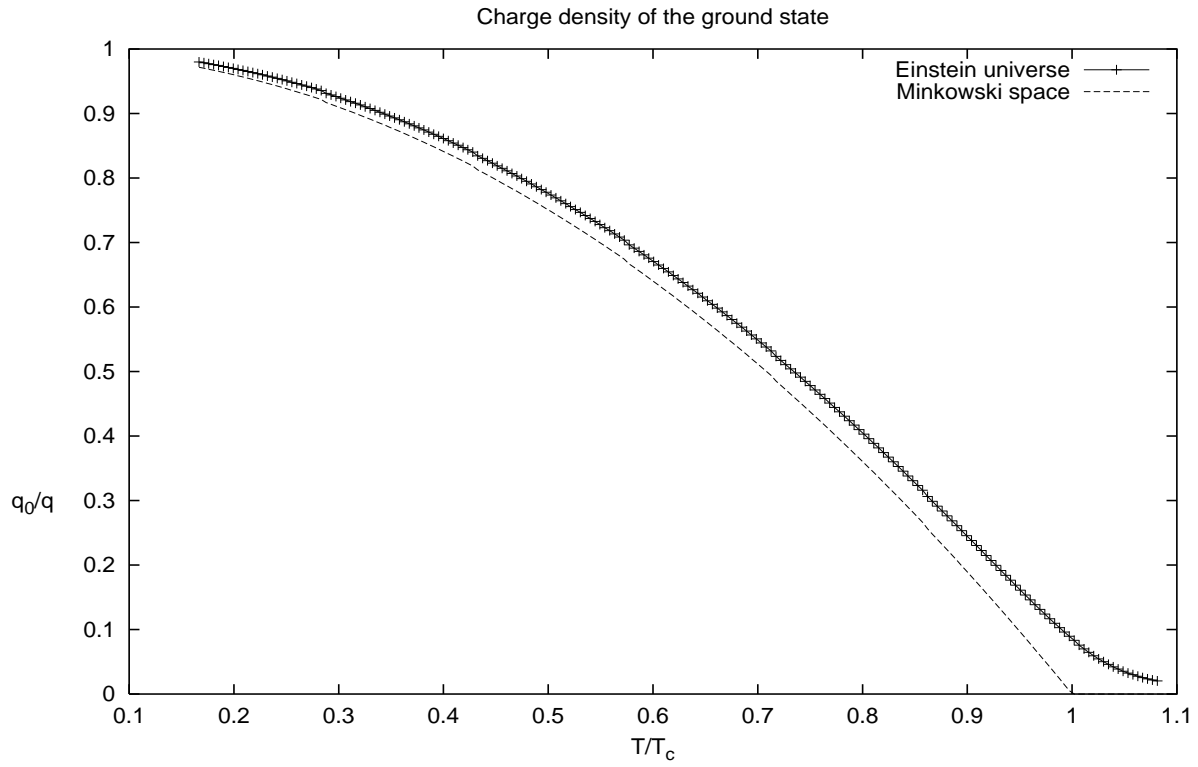

Fig. 1. Charge density of the ground state $q_{0}$ for a closed Robertson-Walker spacetime on a Cauchy surface, where $R=10$ (thick line) compared with the system in the thermodynamic limit, i.e. Minkowski spacetime (thin line) and $Q=0.01$.

The critical temperature $T_{c}$ of Minkowski spacetime, given by

$$
T_{c}=\sqrt{3|q| / m}
$$

(see Haber and Weldon [9]), serves as a useful reference point. Using the fact that $R T=: C=$ const. (proved in $[1,2]$ ), we can define a critical radius $R_{c}$, instead of the critical temperature, given by

$$
R_{c}=\frac{3|Q|}{2 \pi^{2} m C^{2}}
$$

where $Q=q V=q 2 \pi^{2} R^{3}$ is the total charge. This was already realized by Parker and Zhang [5]. Below this radius, the gas is in a condensed state. If the radius becomes larger than the critical radius, the condensate disappears.

The question arises, whether, due to the finite size effects, there is a considerable charge density of the ground state at the critical radius and whether the lifetime of the condensate is considerably longer than indicated by $R_{c}$.

For $T=T_{c}$, we have $y \operatorname{coth} y=0$ (see eq. (2)), i.e. $y^{2}=-\pi^{2}$, and since $y=\pi R \sqrt{\tilde{m}^{2}-\mu^{2}}, \mu=\sqrt{m^{2}-3 /\left(4 R_{c}^{2}\right)}$. This leads to 


$$
\begin{aligned}
\frac{\left|q_{0}\right|}{|q|} & =\frac{1}{|q|} \frac{T_{c}}{R_{c}} \frac{\mu}{\pi^{2}+y^{2}}=\sqrt{\frac{3}{m|q| R_{c}^{2}}} \frac{\sqrt{m^{2}-3 /\left(4 R_{c}^{2}\right)}}{3 \pi^{2} / 4}= \\
& =\sqrt{\frac{3 V}{m|Q| R_{c}^{2}}} \frac{4}{\pi^{2}} \sqrt{m^{2}-3 /\left(4 R_{c}^{2}\right)}= \\
& =\sqrt{\frac{2 \pi^{2} R_{c}}{3 m|Q|} \sqrt{m^{2}-3 /\left(4 R_{c}^{2}\right)}}= \\
& =\frac{4}{m \pi^{2} C} \sqrt{m^{2}-3 /\left(4 R_{c}^{2}\right)} \approx \\
& \approx \frac{4}{\pi^{2} C} .
\end{aligned}
$$

We see that the charge density in the ground state mainly depends on $C$, the product of $R$ and $T$, which is constant as long as the gas can be considered as relativistic. Since this is a large value in the early universe, the finite size effects do not play a considerable role.

The critical radius defined in eq. (3), below which the Bose gas is in a condensed state, makes it possible to think of the following scenario. If there is an abundance of one charge (not necessarily the electric charge) in the early universe, the Bose gas is in a condensed state. The condensate disappears if the radius is larger than the critical radius and the Bose gas is in its usual state. We are not aware of any consequences, a condensed Bose gas would have for the early evolution of the universe, but we think it is worth investigating such consequences.

We give an approximative value for the electric charge (see also [5]). Results of redshift observations [10] show that the present scale factor $R_{0} \geq 10^{27} \mathrm{~cm} \approx$ $10^{41} \mathrm{GeV}^{-1}$ and results for an upper bound on the present charge density [11] give $q_{p}<10^{-24} \mathrm{~cm}^{-3} \approx 10^{-65} \mathrm{GeV}^{3}$. Using a value $C \approx 10^{28}$ and $m=m_{\pi}$, the pion mass, we find

$$
R_{c} \approx 10^{5} \mathrm{GeV}^{-1} \approx 10^{-9} \mathrm{~cm}
$$

We haven taken the maximal value of the electric charge density and the minimal value for the present scale parameter. So the value may differ in both directions considerably. This may be taken as a challenge to find more accurate bounds, especially for the present charge density. In this context it may also be interesting to find bounds for the charge density of other charges. Nevertheless even if the universe is neutral with respect to all charges in question, there may be regions with positive charge density as well as other regions with a negative one, so that condensation in special regions occurs. 


\section{Conclusions}

We analyzed the possibility of Bose-Einstein condensation of a free relativistic charged Bose gas on closed Robertson-Walker spacetimes, described by an adiabatic KMS state. Macroscopic occupation of the ground state sets in, if the temperature is in the region of the critical temperature of a charged relativistic Bose gas on Minkowski spacetime. We have shown the effects due to the finiteness of the spatial volume of closed Robertson-Walker spacetimes but also that they are not relevant in the early universe. Furthermore a critical radius can be defined, above which the condensate disappears. This critical radius depends only on the total charge and the constant product of the temperature and the scale parameter in the Robertson-Walker metric. We also pointed out that this critical radius may have some relevance in cosmology. A next step could be to consider a self-interacting charged scalar field. From a cosmological point of view it may also be interesting to consider the consequences of a condensed Bose gas for the evolution of the universe. Furthermore there is a challenge to find more accurate bounds on the charge density of charged bosons.

\section{References}

[1] M. Trucks, M. Keyl, The free Bose gas on Robertson-Walker spacetimes, Phys. Lett. B 399 (1997) 223-226.

[2] M. Trucks, A KMS-like state of Hadamard type on Robertson-Walker spacetimes and its time evolution, Commun. Math. Phys. 197 (1998) 387-404, gr-qc/9709019.

[3] R. Wald, Quantum Field Theory in Curved Spacetime and Black Hole Thermodynamics (University of Chicago Press, 1994).

[4] S. Singh, R. Pathria, Bose-Einstein condensation in an Einstein universe, J. Phys. A: Math. Gen. 17 (1984) 2982-2994.

[5] L. Parker, Y. Zhang, Ultrarelativistic Bose-Einstein condensation in the Einstein universe and energy conditions, Phys. Rev. D 44 (1991) 2421-2431.

[6] I. Gradshteyn, I. Ryzhik, Table of Integrals, Series and Products (Academic Press, 1980).

[7] A. Erdeleyi et al., Higher Transcendental Functions Vol. 2 (Mc-Graw Hill, 1953).

[8] S. Singh, P. Pandita, Scaling and universality of thermodynamics and correlations of an ideal relativistic Bose gas with pair production, Phys. Rev. A 28 (1983) 1752-1761. 
[9] H. Haber, H. Weldon, Thermodynamics of an ultrarelativistic Bose gas, Phys. Rev. Lett. 46 (1981) 1497-1500.

[10] I. Segal, Mon. N. Roy. Astron. Soc. 242 (1990) 423.

[11] A. Dolgov, Y. Zeldovich, Cosmology and elementary particles, Rev. Mod. Phys. 53 (1981) 1-41. 